\title{
LDL from obese patients with the metabolic syndrome show increased lipid peroxidation and activate platelets
}

\author{
R. Colas • A. Sassolas • M. Guichardant • \\ C. Cugnet-Anceau $\cdot$ M. Moret $\cdot$ P. Moulin • \\ M. Lagarde • C. Calzada
}

Received: 8 June 2011 / Accepted: 14 July 2011 /Published online: 17 August 2011

(C) Springer-Verlag 2011

\begin{abstract}
Aims/hypothesis This study assessed oxidative stress in LDL from obese patients with the metabolic syndrome and compared it with that in LDL from type 2 diabetic patients or control volunteers. It also determined the effect on platelets of LDL from the three groups.

Methods The profiles of lipids, fatty acids and fatty acid oxidation products were determined in LDL isolated from plasma of patients with the metabolic syndrome, patients with type 2 diabetes and volunteers ( $n=10$ per group). The effects of LDL from the participant groups on the platelet arachidonic acid signalling cascade and aggregation were investigated.

Results Compared with LDL from control volunteers, LDL from obese metabolic syndrome and type 2 diabetic patients had lower cholesteryl ester, higher triacylglycerol and lower ethanolamine plasmalogen levels. Proportions of linoleic acid were decreased in phosphatidylcholine and cholesteryl esters in LDL from both patient groups. Among the markers of lipid peroxidation, oxidation products of linoleic acid (hydroxy-octadecadienoic acids) and malondialdehyde were increased by $59 \%$ and twofold, respectively in LDL
\end{abstract}

Electronic supplementary material The online version of this article (doi:10.1007/s00125-011-2272-8) contains peer-reviewed but unedited supplementary material, which is available to authorised users.

R. Colas $\cdot$ A. Sassolas $\cdot$ M. Guichardant $\cdot$ P. Moulin $\cdot$

M. Lagarde $\cdot$ C. Calzada $(\triangle)$

UMR INSERM 1060/Université de Lyon, Cardiovasculaire,

Métabolisme, Diabétologie, et Nutrition (CarMeN), INSA-Lyon,

IMBL, Bât. Louis Pasteur, 20 av. Albert Einstein,

69621 Villeurbanne, France

e-mail: Catherine.Calzada@insa-lyon.fr

A. Sassolas $\cdot$ C. Cugnet-Anceau $\cdot$ M. Moret $\cdot$ P. Moulin Fédération d'endocrinologie, Hospices Civils de Lyon, Bron, France from metabolic syndrome and type 2 diabetic patients. LDL from metabolic syndrome and type 2 diabetic patients were equally potent in activating the platelet arachidonic acid signalling cascade through increased phosphorylation of p38 mitogen-activated protein kinase and cytosolic phospholipase $A_{2}$, and through increased thromboxane $B_{2}$ formation. LDL from patients with the metabolic syndrome and type 2 diabetes potentiated platelet aggregation by threefold and 3.5-fold respectively, whereas control LDL had no activating effects on platelets.

Conclusions/interpretation The metabolic syndrome in obese patients, without or with diabetes, is associated with increased oxidative stress in LDL, which triggers platelet activation.

Trial registration: ClinicalTrials.gov NCT00932087

Funding: The study was funded by INSERM (Institut National de la Santé et de la Recherche Médicale) and ANR (Agence Nationale de la Recherche).

Keywords Fatty acids $\cdot$ LDL $\cdot$ Lipid peroxidation . Metabolic syndrome - Obesity · Oxidised LDL · Platelets . Type 2 diabetes
Abbreviations
cPLA $_{2}$ Cytosolic phospholipase $\mathrm{A}_{2}$
CRP C-reactive protein
HETE Hydroxy-eicosatetraenoic acid
HODE Hydroxy-octadecadienoic acid
MAPK Mitogen-activated protein kinase
MUFA Mono-unsaturated fatty acids
PLSD Protected least significant difference
PUFA Polyunsaturated fatty acids
SFA Saturated fatty acids
$\mathrm{sPLA}_{2} \quad$ Secretory phospholipase $\mathrm{A}_{2}$
$\mathrm{TxB}_{2} \quad$ Thromboxane $\mathrm{B}_{2}$ 


\section{Introduction}

There is strong evidence that type 2 diabetes is associated with increased oxidative stress [1], which may contribute to the elevated risk of atherothrombosis. Oxidatively modified LDL are implicated in the pathogenesis of atherosclerosis and could be present in plasma from type 2 diabetic patients. On the one hand, autoantibodies to oxidised LDL were found to be increased in type 2 diabetic patients with macrovascular disease [2]. On the other hand, we recently showed that LDL from type 2 diabetic patients contained increased levels of lipid peroxides [3]. There is controversy on the occurrence of oxidative stress in patients with the metabolic syndrome. Some studies have shown higher levels of circulating oxidised LDL [4-6] and isoprostanes [7] in individuals with the metabolic syndrome. Other studies show no difference in oxidised LDL levels [8] or markers of oxidative damage (plasma $\mathrm{F}_{2}$-isoprostanes, hydroxy-eicosatetraenoic acid [HETE]) [9] between patients with the metabolic syndrome and healthy individuals. In addition, most studies have focused on lipid peroxides issued from the non-enzymatic peroxidation of arachidonic acid [7,9]. Paradoxically, no data (to our knowledge) are available on circulating lipid peroxides issued from linoleic acid, although it is the main polyunsaturated fatty acid (PUFA) in LDL. Considering that the metabolic syndrome increases the risk of developing type 2 diabetes and cardiovascular diseases $[10,11]$, and that oxidative stress plays an important role in type 2 diabetes, it is important to determine whether oxidised LDL are already present in plasma from patients with the metabolic syndrome.

Increased platelet activation occurs in type 2 diabetes [12] and the metabolic syndrome [13, 14], and may represent a key contributing factor in the process of atherosclerosis and its thrombotic complications. Oxidative stress has been identified as one of the factors closely associated with platelet hyperactivation in diabetes [12] and lipid hydroperoxides have been shown to stimulate platelet aggregation $[15,16]$. In vitro, moreover, oxidised LDL [17, 18] and LDL from patients with type 1 [19] or type 2 diabetes [20] activated platelets via an increase of $\mathrm{p} 38$ mitogen-activated protein kinase (MAPK) phosphorylation and thromboxane $\mathrm{B}_{2}$ $\left(\mathrm{TxB}_{2}\right)$ formation. To date, no data are available on how LDL from patients with the metabolic syndrome interact with platelets.

The objectives of the present study were: (1) to measure and compare different markers of oxidative stress in LDL from obese patients with the metabolic syndrome or type 2 diabetes, and from control volunteers; and (2) to determine the effects of these LDL on platelets.

\section{Methods}

Patients and control volunteers We recruited 30 men (3075 years old) from the Department of Endocrinology and Metabolic Diseases (Hospices Civils de Lyon). Exclusion criteria were smoking, alcohol consumption $>2$ units/day $(20 \mathrm{ml})$, vitamin supplementation and evidence of cardiovascular complications. Patients with the metabolic syndrome were selected according to the criteria of the American Heart Association [21] and had to fulfil three or more of the following factors: waist circumference $>102 \mathrm{~cm}$, triacylglycerol $>1.7 \mathrm{mmol} / 1$, HDLcholesterol $<1 \mathrm{mmol} / \mathrm{l}$, blood pressure $>130 / 85 \mathrm{mmHg}$ and fasting plasma glucose $>5.6 \mathrm{mmol} / \mathrm{l}$. Type 2 diabetic patients had to present with $\mathrm{HbA}_{1 \mathrm{c}}$ greater than $7 \%$ $(53 \mathrm{mmol} / \mathrm{mol})$ and an associated feature of the metabolic syndrome. Patients treated with glitazone drugs were excluded and lipid-lowering drugs were suspended for at least 7 days before venepuncture. Control volunteers were in good health as assessed by medical history and had all above indices in the expected normal range. All participants gave written informed consent. The study protocol was approved by the Local Ethics Committee (CCP Sud Est IV, Hospices Civils de Lyon) and was in accordance with the principles of the Helsinki declaration.

Blood collection Venous blood was collected on EDTA. Plasma was immediately separated by centrifugation at $1,500 \mathrm{~g}$ for $10 \mathrm{~min}$ and frozen at $-80^{\circ} \mathrm{C}$ under nitrogen in the presence of $10 \mu \mathrm{mol} / \mathrm{l}$ butylhydroxytoluene.

Plasma secretory phospholipase $A_{2}$ activity Plasma secretory phospholipase $\mathrm{A}_{2}\left(\mathrm{sPLA}_{2}\right)$ activity was measured with a selective fluorescent substrate (Aterovax, Paris, France). Results are expressed in $\mathrm{U} / \mathrm{ml}$ of sample, with 1 unit defined as the amount of sPLA $\mathrm{P}_{2}$ enzyme that catalyses the release of $1 \mathrm{nmol}$ of product in $1 \mathrm{~min}$. The average intraassay $\mathrm{CV}$ for individual human plasma samples was $8.46 \%$ and the average inter-assay CV was $5.24 \%$. The minimum detectable activity level was $10 \mathrm{U} / \mathrm{ml}$.

LDL isolation LDL (1.019-1.063 $\mathrm{g} / \mathrm{ml})$ were isolated from plasma by potassium bromide stepwise ultracentrifugation $(200,000 \mathrm{~g})$ [22]. LDL preparations were extensively dialysed against PBS containing $1 \mathrm{mmol} / \mathrm{l}$ EDTA. LDL used to determine interactions with platelets were dialysed in a final step against EDTA-free PBS. The concentration of proteins was estimated using a Bradford assay [23]. Enzymatic determinations of total cholesterol and triacylglycerol were processed using commercial kits (Biomérieux, Marcy l'Etoile, France). Plasma highsensitive C-reactive protein (CRP) was measured by nephelometry. LDL particle diameters were determined 
by electrophoresis of plasma in non-denaturating polyacrylamide gradient gels (Lara-Spiral, Couternon, France).

Fatty acid composition of lipid classes Following the addition of internal standards and extraction with ethanol/chloroform $(1: 2$, vol./vol. $)$ in the presence of butylhydroxytoluene $(50 \mu \mathrm{mol} / \mathrm{l})$, lipid classes were separated by thin-layer chromatography. Chloroform/ methanol $(80: 8$, vol./vol.) was used to separate neutral lipids from phospholipids, and chloroform/methanol/ water (63:27:4 by vol.) to separate phosphatidylcholines, phosphatidylethanolamines and sphingomyelins. Neutral lipids were separated into triacylglycerol and cholesteryl esters using hexane/diethylether/acetic acid (80:20:1 by vol.). The different spots were scraped off and treated for 90 min with trifluoride boron:methanol (1:1, vol./vol.) at $100^{\circ} \mathrm{C}$. The derivatised fatty acid methyl esters and fatty dimethylacetals were extracted twice with isooctane and separated by gas chromatography using a gas chromatograph (HP 6890; Agilent Technologies, Massy, France) equipped with a capillary column $(0.25 \mu \mathrm{m}, 30 \mathrm{~m} \times$ $0.25 \mathrm{~mm}$, SP 2380; Supelco, Bellefonte, PA, USA) and a flame ionisation detector. The respective concentrations of fatty acids, quantified according to the amount of appropriate internal standard, were used to calculate the concentrations of lipid classes.

Quantification of total monohydroxylated fatty acids Following lipid extraction, dried extracts were hydrolysed with $10 \mathrm{~mol} / 1 \mathrm{KOH}$ for $20 \mathrm{~min}$ at $60^{\circ} \mathrm{C}$ [24]. Nonesterified hydroxylated fatty acids and fatty acids were first separated on a cartridge column (Oasis Sep-Pak; Waters, Milford, MA, USA) [3]. Non-esterified hydroxylated fatty acids were then separated by thin-layer chromatography with hexane/diethylether/acetic acid (60:40:1 by vol.), extracted with methanol, separated by reversed phase HPLC on an $\mathrm{X}$ Bridge $\mathrm{C}_{18}$ column $(3.5 \mu \mathrm{m}, 4.6 \times 150 \mathrm{~mm}$; Waters) using a gradient solvent of acetonitrile and water $(\mathrm{pH} 3)$, and detected at $235 \mathrm{~nm}$ (for details, see profile in electronic supplementary material [ESM] Fig. 1).

Malondialdehyde determination LDL samples were mixed with thiobarbituric acid $(10 \mathrm{mmol} / \mathrm{l})$, acetic acid and butylhydroxytoluene $(5 \mathrm{mmol} / \mathrm{l})$, and incubated at $95^{\circ} \mathrm{C}$ for $60 \mathrm{~min}$ [25]. The thiobarbituric acid-malondialdehyde adducts were extracted with ethyl acetate, separated on to a Nucleosil $\mathrm{C}_{18}$ column $(5 \mu \mathrm{m}, 4.6 \times 250 \mathrm{~mm}$; MachereyNagel, Düren, Germany) and detected fluorimetrically (excitation $515 \mathrm{~nm}$, emission $553 \mathrm{~nm}$ ).

Vitamin E determination LDL samples, containing tocol as an internal standard, were extracted twice with hexane (4 vol.) following the addition of ethanol (1 vol.) [20]. Tocopherol isomers were separated by reversed phase HPLC on to a Nucleosil $\mathrm{C}_{18}$ column $(5 \mu \mathrm{m}, 4 \times 150 \mathrm{~mm})$ and detected fluorimetrically (excitation $295 \mathrm{~nm}$, emission $340 \mathrm{~nm}$ ).

Platelet isolation and incubation with LDL Blood was collected at the blood bank from healthy volunteers who had not ingested any aspirin or other non-steroidal antiinflammatory drugs in the previous 10 days. Platelets were isolated [26] and incubated for $2 \mathrm{~h}$ at $37^{\circ} \mathrm{C}$ in the presence or absence of LDL $(1 \mathrm{mg} / \mathrm{ml})$.

Platelet p38 MAPK and cytosolic phospholipase $A_{2}$ activation Following platelet lysis, proteins $(30 \mu \mathrm{g})$ were denatured, electrophoresed in 12\% (wt/vol.) Bis-Tris gels (Bio-Rad Laboratories, Marnes-la-Coquette, France) and transferred to nitrocellulose membranes. The membranes were incubated with anti-p38 MAPK or antiphospho-p38 MAPK or anti-phospho-cytosolic phospholipase $\mathrm{A}_{2}$ $\left(\mathrm{CPLA}_{2}\right)$ polyclonal antibodies $(1: 5,000$ for all; Cell Signaling Technologies, Beverly, MA, USA), washed and then incubated with 1:10,000 goat anti-rabbit horseradish peroxidase conjugate. p38 MAPK, phospho-p38 MAPK and phospho-cPLA $A_{2}$ were visualised by enhanced chemiluminescence and bands were quantified by densitometry (Amersham Biosciences, Little Chalfont, UK).

$T x B_{2}$ measurement Platelet $\mathrm{TxB}_{2}$ was quantified by competitive enzyme immunoassay (Amersham Biosciences).

Platelet aggregation Aggregation was measured in isolated platelets in a dual-channel aggregometer (Chrono-log; Coulter, Margency, France) according to the method of Born [27]. Platelet suspensions were pre-incubated for $5 \mathrm{~min}$ at $37^{\circ} \mathrm{C}$ in the presence or absence of LDL $(0.1 \mathrm{mg} / \mathrm{ml})$ and stimulated with sub-threshold concentrations of collagen $(75 \pm 9 \mathrm{ng} / \mathrm{ml})$ while being continuously stirred at 1,000 rpm. The sub-threshold concentration of collagen was defined as the highest concentration of collagen that induced a less than $15 \%$ increase in light transmission. The extent of platelet aggregation was expressed in terms of percentage of change in light transmission at $4 \mathrm{~min}$ after the addition of collagen.

Statistical analysis Results are expressed as the mean \pm SEM. Comparisons between groups were performed using ANOVA followed by Fisher's protected least significant difference (PLSD) post hoc test. Correlation coefficients were determined using linear regression analysis. With a statistical significance of $p<0.05$ and $90 \%$ power, a minimum of eight volunteers per group was required to detect a $50 \%$ difference in the concentration of malondialdehyde in LDL. 


\section{Results}

Clinical characteristics of obese patients with the metabolic syndrome or diabetes and of control volunteers The clinical and metabolic characteristics of the participants are summarised in Table 1. Patients with the metabolic syndrome fulfilled the criteria of the American Heart Association [21]. They were overweight or obese, had abdominal obesity, mild hypertriacylglycerolaemia with low HDL-cholesterol, and were hypertensive. Of ten metabolic syndrome patients, two were being treated against hypertension, whereas four of the ten type 2 diabetic patients were taking antihypertensive medications. This could explain why mean systolic and diastolic blood pressure were higher in the metabolic syndrome than in the type 2 diabetes group. In addition, metabolic syndrome patients showed elevated fasting insulin concentrations and a tendency to have an increased HOMA index, both suggestive of insulin resistance. Levels of high-sensitivity CRP, a circulating marker of systemic inflammation, were increased to similar degrees in metabolic syndrome and type 2 diabetic patients. The type 2 diabetic patients were poorly controlled and also had features of the metabolic syndrome. LDL-cholesterol levels were lower in patients with the metabolic syndrome or type 2 diabetes than in control volunteers, while plasma triacylglycerol levels were higher. Healthy control volunteers had all above indices in the expected normal range.

$\mathrm{sPLA}_{2}$ activity was significantly increased in plasma from patients with the metabolic syndrome or type 2 diabetes (45.7 \pm 4.3 and $51.9 \pm 5.1 \mathrm{U} / \mathrm{ml}$, respectively), compared with plasma from healthy volunteers $(34.4 \pm$ $2.5 \mathrm{U} / \mathrm{ml})$. sPLA $_{2}$ activity showed positive correlations with waist circumference $\left(r^{2}=0.41, p<0.001\right)$ and malondialdehyde concentrations in LDL $\left(r^{2}=0.14, p<0.05\right)$, and a negative correlation with plasmalogen proportions $\left(r^{2}=0.15, p<0.05\right)$.

Lipid and fatty acid composition of LDL from obese patients with the metabolic syndrome or diabetes, compared with that of $L D L$ from control volunteers As shown in Table 2, the concentrations of cholesteryl esters were lower in LDL from both patient groups $(-13 \%$ in metabolic syndrome, $-20 \%$ in type 2 diabetic patients) than in LDL from control volunteers. By contrast, concentrations of triacylglycerol were higher in LDL from metabolic syndrome (50\%) and type 2 diabetic (72\%) patients. No differences in glycerophospholipid concentrations (phosphatidylcholine and phosphatidylethanolamine) were observed between control and the two patient groups. LDL particle sizes were significantly lower in patients with the metabolic syndrome or type 2 diabetes than in control volunteers.
Table 1 Clinical and metabolic characteristics of control volunteers and obese patients with the metabolic syndrome or type 2 diabetes

Data are means \pm SEM $(n=8$ to 10 participants per group)

Hypolipidaemic treatment was interrupted 7 days before inclusion in study; the HOMA index was calculated as: fasting insulin $(\mathrm{pmol} / \mathrm{l}) \times$ fasting glucose $(\mathrm{mmol} / \mathrm{l}) / 400$

${ }^{*} p<0.05$ and $* * p<0.01$ vs LDL from control volunteers; ${ }^{\dagger} p<0.05$ vs LDL from metabolic syndrome patients ( $p$ values obtained by ANOVA followed by Fisher's PLSD post hoc test)

\begin{tabular}{|c|c|c|c|}
\hline Variable & Control & Metabolic syndrome & Type 2 diabetes \\
\hline$n$ & 10 & 10 & 10 \\
\hline Sex & Male & Male & Male \\
\hline Age (years) & $50 \pm 3$ & $58 \pm 2 *$ & $57 \pm 3$ \\
\hline BMI $\left(\mathrm{kg} / \mathrm{m}^{2}\right)$ & $24.3 \pm 0.7$ & $30.7 \pm 1.4^{*}$ & $30.8 \pm 1.1 * *$ \\
\hline Waist circumference $(\mathrm{cm})$ & $92 \pm 2$ & $111 \pm 3^{* *}$ & $116 \pm 7 * *$ \\
\hline Fasting glucose (mmol/l) & $5.5 \pm 0.2$ & $5.6 \pm 0.1$ & $9.0 \pm 1.0 * * \dagger$ \\
\hline Fasting insulin (pmol/1) & $41.1 \pm 6.9$ & $68.4 \pm 12.7^{*}$ & $45.4 \pm 12$ \\
\hline HOMA index & $0.53 \pm 0.13$ & $0.99 \pm 0.20$ & $1.07 \pm 0.36$ \\
\hline CRP (mg/l) & $0.54 \pm 0.12$ & $3.61 \pm 0.82 *$ & $4.82 \pm 1.52 * *$ \\
\hline $\mathrm{HbA}_{1 \mathrm{c}}(\%)$ & $5.4 \pm 0.1$ & $5.7 \pm 0.1$ & $10.4 \pm 0.9 * * \dagger$ \\
\hline $\mathrm{HbA}_{1}(\mathrm{mmol} / \mathrm{mol})$ & $35.5 \pm 0.6$ & $38.5 \pm 1.4$ & $90.2 \pm 9.8 * * \dagger$ \\
\hline Systolic BP (mmHg) & $121 \pm 3$ & $144 \pm 3 * *$ & $132 \pm 6^{* \dagger}$ \\
\hline Diastolic BP (mmHg) & $78 \pm 4$ & $88 \pm 5^{*}$ & $77 \pm 3 \dagger$ \\
\hline Total cholesterol (mmol/l) & $6.0 \pm 0.4$ & $5.2 \pm 0.3$ & $4.8 \pm 0.4^{*}$ \\
\hline HDL-cholesterol (mmol/1) & $1.4 \pm 0.1$ & $1.1 \pm 0.1 * *$ & $0.9 \pm 0.1^{* *}$ \\
\hline Total cholesterol:HDL-cholesterol & $4.5 \pm 0.3$ & $4.9 \pm 0.3$ & $5.3 \pm 0.3^{*}$ \\
\hline LDL-cholesterol (mmol/1) & $4.1 \pm 0.4$ & $3.2 \pm 0.2$ & $2.7 \pm 0.4^{* *}$ \\
\hline VLDL-cholesterol (mmol/l) & $0.5 \pm 0.1$ & $0.8 \pm 0.1 * *$ & $1.0 \pm 0.1 * * \dagger$ \\
\hline Triacylglycerol (mmol/l) & $1.3 \pm 0.1$ & $2.2 \pm 0.3 * *$ & $2.6 \pm 0.2 * *$ \\
\hline Glucose-lowering treatment $(\%)$ & 0 & 0 & 80 \\
\hline Antihypertensive treatment $(\%)$ & 0 & 20 & 40 \\
\hline Hypolipidaemic treatment (\%) & 0 & 10 & 20 \\
\hline
\end{tabular}


Table 2 Lipid classes and particle size of LDL from control volunteers and obese patients with the metabolic syndrome or type 2 diabetes

\begin{tabular}{lccc}
\hline Variable & Control & Metabolic syndrome & Type 2 diabetes \\
\hline Cholesteryl esters (nmol/mg protein) & $2,459 \pm 118$ & $2,132 \pm 61^{* *}$ & $1,975 \pm 88^{* *}$ \\
Phosphatidylcholines (nmol/mg protein) & $521 \pm 17$ & $477 \pm 18$ & $479 \pm 21$ \\
Phosphatidylethanolamines (nmol/mg protein) & $17 \pm 2$ & $15 \pm 1$ & $18 \pm 1$ \\
Triacylglycerol (nmol/mg protein) & $140 \pm 11$ & $210 \pm 19^{*}$ & $241 \pm 37^{* *}$ \\
LDL particle size (nm) & $25.1 \pm 0.2$ & $24.4 \pm 0.2 *$ & $24.4 \pm 0.1 *$ \\
\hline
\end{tabular}

Data are means $\pm \operatorname{SEM}(n=8$ to 10 participants per group)

$* p<0.05$ and $* * p<0.01$ vs LDL from control volunteers ( $p$ values obtained by ANOVA followed by Fisher's PLSD post hoc test)

The fatty acid composition of cholesteryl esters and phosphatidylcholine differed between patients and control volunteers (Table 3 ). The proportions of PUFA decreased in cholesteryl esters from metabolic syndrome patients, while saturated fatty acids (SFA) and mono-unsaturated fatty acids (MUFA) proportions increased. In particular, the proportion of linoleic acid (18:2 n-6), the main PUFA, decreased in cholesteryl esters and phosphatidylcholine from metabolic syndrome patients. Although palmitoleic acid (16:1 n-7) represents a minor fatty acid, its proportion increased by $70 \%$ in cholesteryl esters from that group. Concerning LDL from type 2 diabetic patients, PUFA proportions decreased while those of SFA increased and
MUFA were unchanged. In particular, the proportions of linoleic acid decreased while those of arachidonic acid (20:4 n-6) increased in cholesteryl esters and phosphatidylcholine from type 2 diabetic LDL.

Special care was taken to measure ethanolamine plasmalogens (or alkenyl,acyl-glycerophosphoethanolamines). They constitute a glycerophospholipid subclass with a 16:0, 18:0 or $18: 1$ fatty chain attached at the $s n-1$ position via a vinyl ether bond, and are more susceptible to oxidation [28]. As shown in Fig. 1a, the proportions of 16:0 dimethyl acetal issued from the alkenyl residue and of 18:0 dimethylacetal were decreased by $22 \%$ and $23 \%$, respectively in LDL from metabolic syndrome patients compared

Table 3 Main fatty acid compositions of cholesteryl esters and phosphatidylcholines in LDL from control volunteers and obese patients with metabolic syndrome or type 2 diabetes

\begin{tabular}{|c|c|c|c|c|c|c|}
\hline \multirow[t]{2}{*}{ Fatty acid (mol\%) } & \multicolumn{3}{|l|}{ Cholesteryl esters } & \multicolumn{3}{|c|}{ Phosphatidylcholines } \\
\hline & Type 2 diabetes & Metabolic syndrome & Control & Type 2 diabetes & Metabolic syndrome & Control \\
\hline 16:0 & $13.7 \pm 0.3^{*}$ & $13.5 \pm 0.4^{*}$ & $12.4 \pm 0.4$ & $34.3 \pm 0.5$ & $34.7 \pm 0.4$ & $33.5 \pm 0.7$ \\
\hline $16: 1 n-7$ & $2.8 \pm 0.3$ & $4.2 \pm 0.3^{* *}$ & $2.5 \pm 0.3$ & $0.3 \pm 0.1$ & $0.5 \pm 0.1$ & $0.3 \pm 0.1$ \\
\hline $16: 1 n-9$ & $0.5 \pm 0.1$ & $0.4 \pm 0.1$ & $0.3 \pm 0.1$ & 0 & 0 & 0 \\
\hline 18:0 & $1.0 \pm 0.1$ & $1.0 \pm 0.1$ & $1.0 \pm 0.1$ & $15.3 \pm 0.7$ & $15.8 \pm 0.7$ & $16.0 \pm 0.5$ \\
\hline $18: 1 n-7$ & $1.0 \pm 0.2$ & $0.7 \pm 0.2$ & $1.0 \pm 0.2$ & $1.2 \pm 0.3$ & $1.0 \pm 0.2$ & $1.3 \pm 0.3$ \\
\hline $18: 1 n-9$ & $22.1 \pm 0.7$ & $21.7 \pm 0.7$ & $20.6 \pm 0.7$ & $11.6 \pm 0.3$ & $10.9 \pm 0.5$ & $10.9 \pm 0.5$ \\
\hline $18: 2 n-6$ & $46.7 \pm 1.1 * *$ & $46.7 \pm 1.4^{* *}$ & $52.0 \pm 1.2$ & $19.6 \pm 0.7^{*}$ & $19.2 \pm 0.7^{*}$ & $21.8 \pm 1.0$ \\
\hline $18: 3 n-3$ & $0.5 \pm 0.1$ & $0.7 \pm 0.0$ & $0.4 \pm 0.1$ & 0 & 0 & 0 \\
\hline $18: 3 n-6$ & $0.8 \pm 0.2$ & $1.1 \pm 0.1$ & $0.8 \pm 0.1$ & 0 & 0 & 0 \\
\hline $20: 3 n-6$ & $0.9 \pm 0.1$ & $0.9 \pm 0.1$ & $0.8 \pm 0.0$ & $3.3 \pm 0.2$ & $3.7 \pm 0.3^{*}$ & $3.1 \pm 0.2$ \\
\hline $20: 4 n-6$ & $8.3 \pm 0.5 * * \dagger$ & $7.0 \pm 0.6$ & $6.2 \pm 0.4$ & $9.3 \pm 0.5^{*}$ & $8.8 \pm 0.7$ & $7.2 \pm 0.6$ \\
\hline $20: 5 n-3$ & $1.0 \pm 0.2$ & $1.3 \pm 0.2$ & $1.3 \pm 0.2$ & $0.9 \pm 0.2$ & $1.1 \pm 0.1$ & $1.1 \pm 0.3$ \\
\hline $22: 5 n-3$ & 0 & 0 & 0 & $0.4 \pm 0.1$ & $0.4 \pm 0.2$ & $0.4 \pm 0.2$ \\
\hline $22: 6 n-3$ & $0.6 \pm 0.1$ & $0.6 \pm 0.1$ & $0.7 \pm 0.1$ & $2.9 \pm 0.2$ & $2.6 \pm 0.3$ & $3.2 \pm 0.4$ \\
\hline SFA & $14.7 \pm 0.3^{*}$ & $14.7 \pm 0.4^{*}$ & $13.5 \pm 0.5$ & $49.9 \pm 0.6$ & $50.9 \pm 0.7$ & $50.0 \pm 0.9$ \\
\hline MUFA & $26.4 \pm 0.5$ & $27.1 \pm 1.0^{*}$ & $24.4 \pm 0.8$ & $13.4 \pm 0.4$ & $13.0 \pm 0.6$ & $12.9 \pm 0.6$ \\
\hline PUFA & $59.0 \pm 0.7^{*}$ & $58.3 \pm 1.2^{*}$ & $62.1 \pm 1.1$ & $36.7 \pm 0.5$ & $36.1 \pm 0.8$ & $37.1 \pm 0.9$ \\
\hline
\end{tabular}

Data are means \pm SEM $(n=8-9$ participants per group)

$*_{p}<0.05$ and $*^{*} p<0.01$ vs LDL from control volunteers; ${ }^{\dagger} p<0.05$ vs LDL from metabolic syndrome patients $(p$ values obtained by ANOVA followed by Fisher's PLSD post hoc test) 


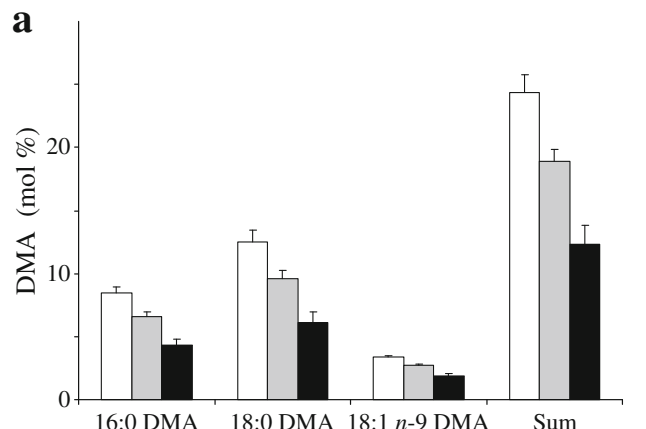

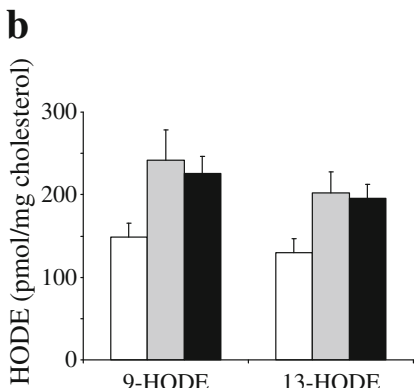

c

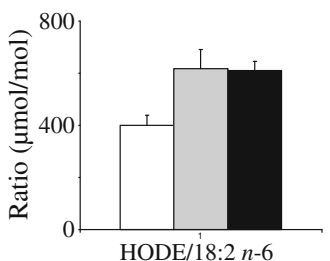

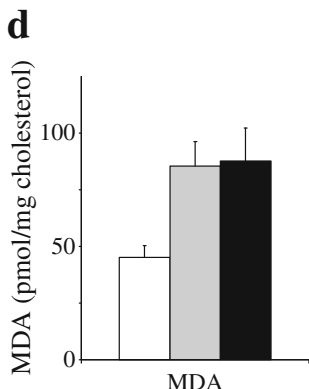

Fig. 1 Indices of lipid peroxidation in LDL from control volunteers (white bars) and obese patients with metabolic syndrome (grey bars) or type 2 diabetes (black bars). a Fatty dimethylacetals (DMA) derived from phosphatidylethanolamine plasmalogens. b 9- and 13HODE, (c) HODE/18:2 n-6 ratio and (d) malondialdehyde (MDA). Data are means \pm SEM ( $n=7-10$ participants per group). $p<0.05$ for 16:0 DMA, 18:0 DMA, DMA sum, 9-HODE, 13-HODE and HODE/ 18:2 $n-6$, and $p<0.01$ for malondialdehyde in LDL from metabolic

with control LDL. In LDL from type 2 diabetic patients, 16:0, 18:0 and 18:1 dimethylacetal species were decreased by $47 \%, 50 \%$ and $42 \%$, respectively. Altogether, the sum of dimethylacetal species, representative of plasmalogens, was decreased by $22 \%$ and $49 \%$ in LDL from patients with the metabolic syndrome or type 2 diabetes, respectively. Interestingly, ethanolamine plasmalogen proportions were negatively correlated with waist circumference $\left(r^{2}=0.22, p<0.01\right)$.

Lipid peroxides and antioxidant contents of $L D L$ from obese patients with the metabolic syndrome or diabetes, compared with those of LDL from control volunteers The concentrations of total (free and esterified) hydroxylated fatty acids, the stable primary products of lipid peroxidation, were determined in LDL from patient and control groups. They mainly consisted of hydroxy-octadecadienoic acids (HODE) derived from linoleic acid and HETE derived from arachidonic acid. Concentrations of 9-HODE and 13HODE increased by $62 \%$ and $55 \%$ respectively in LDL from metabolic syndrome patients and by $52 \%$ and $50 \%$ in LDL from type 2 diabetic patients (Fig. 1b). Additionally, the ratio of total HODE to linoleic acid increased by $54 \%$ and $52 \%$ in LDL from metabolic syndrome and type 2 diabetic patients, respectively (Fig. 1c). Among HETE forms, 8-, 11-, 12- and 15HETE were detected in LDL from each participant. There were no significant differences in the concentrations of HETE between metabolic syndrome patients and control volunteers (data not shown). Only the 15-HETE isomer concentration was significantly increased by $82 \%$ in LDL from type 2 diabetic patients, compared with LDL from control volunteers $(50.8 \pm 7.6 \mathrm{pmol} / \mathrm{mg}$ cholesterol vs $28.0 \pm 4.3 \mathrm{pmol} / \mathrm{mg}$ cholesterol, $p<0.05)$. Concentra- syndrome patients vs LDL from control volunteers; $p<0.05$ for 9-HODE, 13-HODE and HODE/18:2 $n$-6, and $p<0.01$ for 16:0 DMA, 18:0 DMA, 18:1 n-9 DMA, DMA sum and malondialdehyde in LDL from type 2 diabetic patients vs LDL from control volunteers; $p<0.05$ for 16:0 DMA, 18:0 DMA, 18:1 n-9 DMA and DMA sum in LDL from type 2 diabetic patients vs LDL from metabolic syndrome patients. $p$ values were obtained by ANOVA followed by Fisher's PLSD post hoc test

tions of malondialdehyde, issued from the degradation of fatty acid hydroperoxides, were significantly increased by twofold in patients with the metabolic syndrome or type 2 diabetes (Fig. 1d). Waist circumference was mildly correlated with triacylglycerol and malondialdehyde levels in LDL $\left(r^{2}=0.13\right.$ and 0.25 , respectively, $\left.p<0.05\right)$ (ESM Fig. 2a, b).

The concentrations of vitamin E, the main liposoluble antioxidant, were determined in LDL. $\alpha$-Tocopherol concentrations did not differ between patients with the metabolic syndrome or type 2 diabetes $(6.7 \pm 0.5$ and $6.0 \pm 0.3 \mathrm{nmol} / \mathrm{mg}$ protein, respectively) vs control volunteers $(5.9 \pm 0.3 \mathrm{nmol} / \mathrm{mg}$ protein). $\gamma$-Tocopherol, which represents $10 \%$ of $\alpha$-tocopherol, was increased in LDL from metabolic syndrome patients $(0.9 \pm 0.1 \mathrm{nmol} / \mathrm{mg}$ protein $)$ compared with LDL from control $(0.6 \pm 0.1 \mathrm{nmol} / \mathrm{mg}$ protein $)$, but did not differ in LDL from type 2 diabetic patients $(0.7 \pm 0.1 \mathrm{nmol} / \mathrm{mg}$ protein $)$.

LDL isolated from obese patients with the metabolic syndrome or diabetes, or from control volunteers: effects on platelet arachidonic acid signalling cascade To determine the effects of LDL on the platelet arachidonic acid cascade, platelets from healthy donors were incubated for $2 \mathrm{~h}$ at $37^{\circ} \mathrm{C}$ in the absence or presence of LDL from both patient groups or control volunteers $(1 \mathrm{mg} / \mathrm{ml})$. Phosphorylated amounts of p38 MAPK and $\mathrm{cPLA}_{2}$, key enzymes involved in the release of arachidonic acid from membrane phospholipids, as well as concentrations of $\mathrm{TxB}_{2}$, the stable catabolite of the pro-aggregatory arachidonic acid metabolite thromboxane $A_{2}$, were determined. As shown in Fig. 2, the addition of LDL from control volunteers to platelets had no effects on p38 MAPK and $\mathrm{cPLA}_{2}$ phosphorylation, compared with platelets alone. By contrast, the addition of 


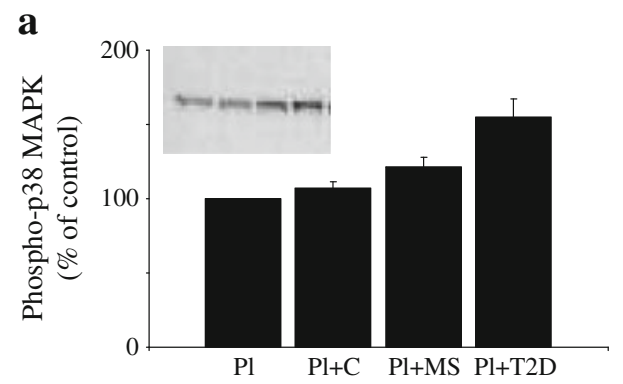

b

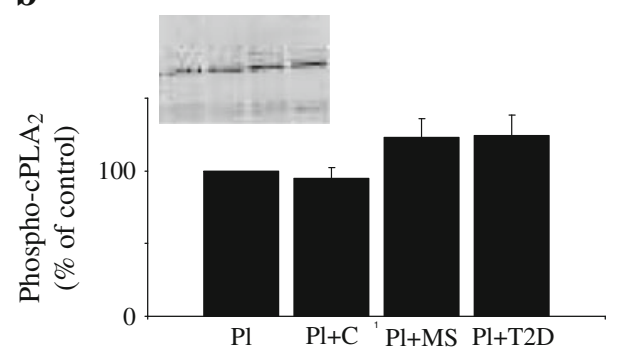

Fig. 2 Effects of LDL from control volunteers and obese patients with metabolic syndrome or type 2 diabetes on the phosphorylation of (a) p38 MAPK and (b) $\mathrm{cPLA}_{2}$ in platelets. Platelets (Pl) from blood donors were incubated for $2 \mathrm{~h}$ at $37^{\circ} \mathrm{C}$ in the absence or presence of LDL (1 $\mathrm{mg}$ protein $/ \mathrm{ml})$ from either control volunteers (C), metabolic syndrome patients (MS) or type 2 diabetic patients (T2D). Representative immunoblots and histograms of the normalised amount of phosphorylation of p38 MAPK and $\mathrm{cPLA}_{2}$ are shown. Data are means $\pm \operatorname{SEM}(n=7-8$ participants per group). $p<0.05$ for phospho-p38 MAPK and phospho-cPLA 2 in Pl+MS vs $\mathrm{Pl}$; $p<0.05$ for phospho-cPLA 2 and $p<0.01$ for phospho-p38 MAPK in $\mathrm{Pl}+\mathrm{T} 2 \mathrm{D}$ vs $\mathrm{Pl} ; p<0.05$ for phospho-p38 MAPK in $\mathrm{Pl}+\mathrm{T} 2 \mathrm{D}$ vs P1+MS. $p$ values were obtained by ANOVA followed by Fisher's PLSD post hoc test

LDL from patients with the metabolic syndrome or type 2 diabetes significantly increased p38 MAPK phosphorylation, by $21 \%$ and $55 \%$ respectively, and cPLA 2 phosphorylation by $23 \%$ and $24 \%$ respectively. Compared with control platelets, the incubation of platelets with LDL from patients with the metabolic syndrome or type 2 diabetes resulted in a twofold increased basal concentration of $\mathrm{TxB}_{2}$, whereas LDL from control volunteers had no effects (Fig. 3). Platelet $\mathrm{TxB}_{2}$ was negatively correlated with plasmalogen proportions $\left(r^{2}=0.44\right)$ and positively with HODE and malondialdehyde concentrations in LDL $\left(r^{2}=0.24\right.$ and $r^{2}=0.31$, respectively, $\left.p<0.01\right)$ (ESM Fig. 2c, d).

Effect on platelet aggregation of $L D L$ isolated from obese patients with the metabolic syndrome or diabetes, or from control volunteers To determine the effect of LDL on platelet aggregation, platelets were pre-incubated for 5 min with low concentrations of LDL $(0.1 \mathrm{mg} / \mathrm{ml})$ and then stimulated with sub-threshold concentrations of collagen for another $4 \mathrm{~min}$. As shown in Fig. 4, LDL

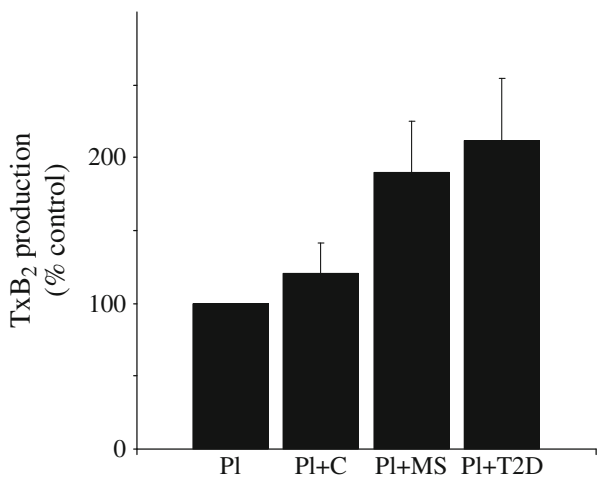

Fig. 3 Effects of LDL from control volunteers and obese patients with metabolic syndrome or type 2 diabetes on $\mathrm{TxB}_{2}$ concentrations in unstimulated platelets. Platelets $(\mathrm{Pl})$ from blood donors were incubated for $2 \mathrm{~h}$ at $37^{\circ} \mathrm{C}$ in the absence or presence of LDL (1 $\mathrm{mg}$ protein $/ \mathrm{ml}$ ) from either control volunteers (C), metabolic syndrome patients (MS) or type 2 diabetic patients (T2D). $\mathrm{TxB}_{2}$ concentrations in control unstimulated platelets were $187 \pm 37 \mathrm{pmol} / 10^{9}$ platelets. Data, expressed as per cent of control, are means \pm SEM ( $n=10$ participants per group). $p<0.05$ for $\mathrm{P} 1+\mathrm{T} 2 \mathrm{D}$ vs $\mathrm{Pl}$ and $\mathrm{Pl}+\mathrm{MS}$ vs Pl. $p$ values were obtained by ANOVA followed by Fisher's PLSD post hoc test

from control volunteers had no effect on collagen-induced platelet aggregation, compared with platelets incubated with buffer. By contrast, pre-incubation of platelets with LDL from metabolic syndrome or type 2 diabetic patients resulted in stimulation of platelet aggregation in response to sub-threshold concentrations of collagen (197\% and $251 \%$, respectively). Platelet aggregation was also potentiated by lower concentrations $(50 \mu \mathrm{g} / \mathrm{ml})$ of LDL from type 2 diabetic patients (data not shown).

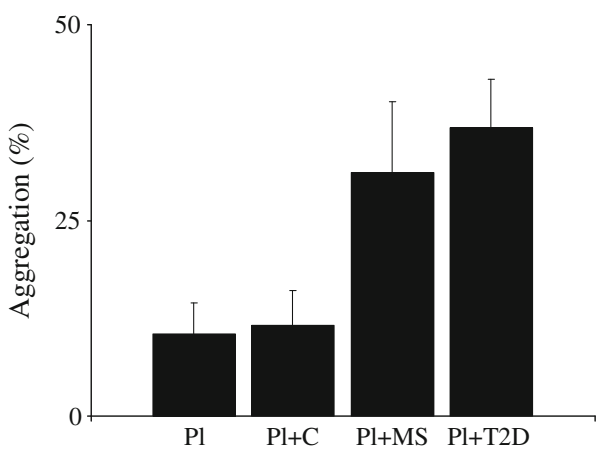

Fig. 4 Effects of LDL from control participants and obese patients with metabolic syndrome or type 2 diabetes on the aggregation of platelets induced by collagen. Platelets $(\mathrm{Pl})$ from blood donors were pre-incubated for $5 \mathrm{~min}$ at $37^{\circ} \mathrm{C}$ in the absence or presence of LDL $(0.1 \mathrm{mg}$ protein $/ \mathrm{ml})$ from either control volunteers $(\mathrm{C})$, metabolic syndrome patients (MS) or type 2 diabetic patients (T2D) and stimulated with sub-threshold concentrations of collagen $(75 \pm 9 \mathrm{ng} / \mathrm{ml})$. Data, expressed as per cent of aggregation, are means \pm SEM of five participants per group. $p<0.05$ for $\mathrm{Pl}+\mathrm{MS}$ vs $\mathrm{Pl} ; p<0.01$ for $\mathrm{P} 1+\mathrm{T} 2 \mathrm{D}$ vs $\mathrm{Pl} . p$ values were obtained by ANOVA followed by Fisher's PLSD post hoc test 


\section{Discussion}

The evidence for increased oxidative stress in patients with the metabolic syndrome is still a matter of debate. In the present study, we provide several lines of evidence for increased oxidative stress in LDL from obese metabolic syndrome patients, as assessed by different complementary indices of oxidative stress and by special focus on oxidation products of linoleic acid, the most abundant PUFA in LDL. Higher concentrations of total HODE and lower proportions of linoleic acid in phosphatidylcholine and cholesteryl esters were found in LDL from metabolic syndrome patients compared with control LDL. Interestingly, HODE and linoleic acid levels were modified to the same extent in LDL from type 2 diabetic patients. Conversely, there were no changes in total HETE concentrations in metabolic syndrome patients, which is in agreement with a recent study showing no differences in the levels of oxidation products of arachidonic acid (total HETE and $\mathrm{F}_{2}$-isoprostanes) in individuals with features of metabolic syndrome [9]. This suggests that oxidation products of linoleic acid could be better markers of lipid peroxidation than those of arachidonic acid. It should be mentioned that participants did not supply a dietary record. Although we are not aware of any supplementation study reporting a concomitant decrease of linoleic acid and increase of arachidonic acid in plasma or LDL, we cannot exclude a potential effect of fat intake on plasma fatty acid composition.

The presence of oxidative stress in LDL from metabolic syndrome patients was confirmed by the decreased concentrations of plasmalogens in LDL from metabolic syndrome patients $(-22 \%)$ as well as in LDL from type 2 diabetic patients $(-49 \%)$, compared with control LDL. This result indicates that plasmalogens may be targets and markers of ongoing oxidative stress, and corroborates previous data showing a $20 \%$ decrease of plasmalogen phosphatidylethanolamine in erythrocyte membranes from hyperlipidaemic patients [29]. In addition, we found that malondialdehyde, a commonly used overall marker of lipid peroxidation that has been described to correlate with total isoprostanes in plasma and tissues [30, 31], was increased to a similar extent in LDL from metabolic syndrome and type 2 diabetic patients, in agreement with the enhanced serum levels of thiobarbituric acid reactive species observed in type 2 diabetic patients [32]. Although isoprostanes are recognised as reliable markers of oxidative stress in vivo, they derive exclusively from the free radical-induced peroxidation of arachidonic acid and their levels were either reported to be increased [7] or unchanged [9] in patients with the metabolic syndrome. Altogether, the increase of several complementary indices of oxidative stress and especially of oxidised products of linoleic acid in LDL from metabolic syndrome patients supports the hypothesis that the metabolic syndrome is associated with higher levels of circulating oxidised LDL [4-6]. In addition, compared with control LDL, LDL from patients with the metabolic syndrome showed higher triacylglycerol and lower cholesteryl ester levels as well as decreased particle size, suggesting a higher percentage of smaller and denser LDL particles. Therefore, our data corroborate reports that the metabolic syndrome is associated with small and dense LDL particles [5, 33, 34], which are known to be more susceptible to oxidative modification [35].

Regarding the main antioxidant in LDL, we found similar concentrations of $\alpha$-tocopherol in patients with the metabolic syndrome or type 2 diabetes, and higher concentrations of $\gamma$-tocopherol in metabolic syndrome patients. There have been discordant reports about vitamin E levels in patients with metabolic syndrome or type 2 diabetes, showing either lower vitamin $\mathrm{E}$ concentrations in participants who had a feature of the metabolic syndrome and also consumed fewer fruits and vegetables [36], or no difference among participants with the metabolic syndrome or type 2 diabetes [37, 38]. Only one study to our knowledge has reported slightly higher serum $\gamma$-tocopherol levels [38] and no changes of $\alpha$-tocopherol among participants with the metabolic syndrome. Our results obtained ex vivo suggest that tocopherols may have been spared by molecules (such as plasmalogens) serving as primary targets of free radicals during LDL oxidation [39].

It is worth noting that oxidative stress was enhanced to a similar extent in LDL from metabolic syndrome and from type 2 diabetic patients, suggesting that factors associated with the metabolic syndrome, rather than poor glycaemic control, are key factors associated with oxidative modification of LDL. In agreement with this statement, our results show correlations between waist circumference and plasmalogens or malondialdehyde in LDL, suggesting an association between an excess of visceral fat and biochemical variables associated with oxidative stress in LDL. It is also likely that obesity tightly associated with the metabolic syndrome may represent a major factor in the oxidative stress seen in LDL from obese metabolic syndrome patients.

Beside the above, our results show that the activity of sPLA $_{2}$ is correlated with plasmalogens and malondialdehyde in LDL, suggesting that this enzyme catalyses the hydrolysis of oxidised phospholipids in LDL [40]. The correlation between the activity of $\mathrm{sPLA}_{2}$ and waist circumference is in line with the association of $\mathrm{SPLA}_{2}$ with the metabolic syndrome [41].

Regarding the functionality of LDL from patients with metabolic syndrome, we show for the first time that LDL from metabolic syndrome patients activate platelets and prime collagen-induced platelet aggregation. The activation occurs through an increased phosphorylation of p38 MAPK, the stress kinase responsible for the phosphorylation of $\mathrm{CPLA}_{2}$ [42], the key enzyme involved in the release of arachidonic acid from membrane phospholipids [43]. It resulted in 
increased formation of thromboxane $\mathrm{A}_{2}$, as assessed by the increased concentration of the stable catabolite, $\mathrm{TxB}_{2}$. We confirm that LDL isolated from type 2 diabetic patients activate platelets through the arachidonic acid cascade [20] and give new evidence for a priming effect of LDL on platelet aggregation, whereas LDL from control volunteers had no activating effects. As for the question of which molecules in LDL from the two patient groups are responsible for platelet activation, our data suggest that lipid peroxides may be implicated. LDL from obese patients with metabolic syndrome or type 2 diabetes showed similarly increased levels of lipid peroxides and activated platelets. In addition, correlation analyses show that the platelet $\mathrm{TxB}_{2}$ concentration is related to concentrations of HODE and malondialdehyde in LDL, suggesting that lipid peroxides could underlie the activating effects of LDL on platelets and that glycation is not necessarily required. In future studies it will be important to take into account obesity in order to identify the mechanism by which oxidised LDL activate platelets.

In addition, our results obtained ex vivo strengthen the notion that fatty acid peroxides are implicated in platelet activation as previously demonstrated in in vitro studies $[15,44]$. Indeed, we have previously shown that fatty acid hydroperoxides, precursors of HETE and HODE, potentiated platelet aggregation and activated platelets via the arachidonic acid signalling cascade [16]. More recently, the Podrez group showed that oxidised choline glycerophospholipids induce platelet aggregation via CD36 [45].

Altogether, our results support the idea that: (1) oxidative stress is increased in LDL from obese metabolic syndrome patients with or without type 2 diabetes; and (2) in vivo oxidatively modified LDL from metabolic syndrome patients activate control platelets to the same extent as LDL from type 2 diabetic patients. Considering that the metabolic syndrome often precedes the occurrence of diabetes [46] and that cardiovascular complications occur frequently at the onset of type 2 diabetes, our data suggest that oxidative stress in metabolic syndrome is an early promoter of premature atherothrombosis [47]. The identification of specific targets of oxidative stress in the metabolic syndrome, such as linoleic acid-containing lipids, might provide opportunities to selectively slow down some specific lipid peroxidation processes in LDL at the stage of the metabolic syndrome and to prevent the associated platelet hyperactivation.

Acknowledgements This study was supported by INSERM and ANR grant (ANR-05-COD-D019-01). R. Colas was funded by the French Ministry of Education and Research. C. Calzada is supported by the CNRS. The authors gratefully thank Z. Mallat and Aterovax (Paris, France) for the analysis of $\mathrm{SPLA}_{2}$ activity. The authors also thank all participants, the nursing staff at Hospices Civils de Lyon for expert blood drawing assistance and M. Broyer for particle size data analysis.
Contribution statement $\mathrm{CC}, \mathrm{RC}, \mathrm{ML}$ and $\mathrm{PM}$ conceived and designed the study. CCA, MM and PM recruited the patients. RC and AS acquired data. All authors interpreted data. CC wrote the manuscript and RC, ML, PM, AS, MG, CCA and MM revised the manuscript critically. All authors approved the final version of the manuscript.

Duality of interest The authors fully declare that there is no duality of interest associated with this manuscript.

\section{References}

1. Stephens JW, Khanolkar MP, Bain SC (2009) The biological relevance and measurement of plasma markers of oxidative stress in diabetes and cardiovascular disease. Atherosclerosis 202:321329

2. Hsu RM, Devaraj S, Jialal I (2002) Autoantibodies to oxidized low-density lipoprotein in patients with type 2 diabetes mellitus. Clin Chim Acta 317:145-150

3. Colas R, Pruneta-Deloche V, Guichardant $M$ et al (2010) Increased lipid peroxidation in LDL from type-2 diabetic patients. Lipids 45:723-731

4. Holvoet P, Kritchevsky SB, Tracy RP et al (2004) The metabolic syndrome, circulating oxidized LDL, and risk of myocardial infarction in well-functioning elderly people in the health, aging, and body composition cohort. Diabetes 53:1068-1073

5. Sigurdardottir V, Fagerberg B, Hulthe J (2002) Circulating oxidized low-density lipoprotein (LDL) is associated with risk factors of the metabolic syndrome and LDL size in clinically healthy 58-year-old men (AIR Study). J Intern Med 252:440447

6. Park SH, Kim JY, Lee JH, Park HY (2011) Elevated oxidized lowdensity lipoprotein concentrations in postmenopausal women with the metabolic syndrome. Clin Chim Acta 412:435-440

7. Hansel B, Giral P, Nobecourt E et al (2004) Metabolic syndrome is associated with elevated oxidative stress and dysfunctional dense high-density lipoprotein particles displaying impaired antioxidative activity. J Clin Endocrinol Metab 89:4963-4971

8. Sjogren P, Basu S, Rosell M et al (2005) Measures of oxidized low-density lipoprotein and oxidative stress are not related and not elevated in otherwise healthy men with the metabolic syndrome. Arterioscler Thromb Vasc Biol 25:2580-2586

9. Seet RC, Lee CY, Lim EC et al (2010) Markers of oxidative damage are not elevated in otherwise healthy individuals with the metabolic syndrome. Diabetes Care 33:1140-1142

10. Wilson PW, D'Agostino RB, Parise H, Sullivan L, Meigs JB (2005) Metabolic syndrome as a precursor of cardiovascular disease and type 2 diabetes mellitus. Circulation 112:3066-3072

11. Gami AS, Witt BJ, Howard DE et al (2007) Metabolic syndrome and risk of incident cardiovascular events and death: a systematic review and meta-analysis of longitudinal studies. J Am Coll Cardiol 49:403-414

12. Vericel E, Januel C, Carreras M, Moulin P, Lagarde M (2004) Diabetic patients without vascular complications display enhanced basal platelet activation and decreased antioxidant status. Diabetes 53:1046-1051

13. Vaduganathan M, Alviar CL, Arikan ME et al (2008) Platelet reactivity and response to aspirin in subjects with the metabolic syndrome. Am Heart J 156:1002 e1001-1002 e1007

14. Vaidya D, Yanek LR, Faraday N, Moy TF, Becker LC, Becker DM (2009) Native platelet aggregation and response to aspirin in persons with the metabolic syndrome and its components. Metab Syndr Relat Disord 7:289-296 
15. Calzada C, Vericel E, Mitel B, Coulon L, Lagarde M (2001) 12(S)Hydroperoxy-eicosatetraenoic acid increases arachidonic acid availability in collagen-primed platelets. J Lipid Res 42:14671473

16. Coulon L, Calzada C, Moulin P, Vericel E, Lagarde M (2003) Activation of p38 mitogen-activated protein kinase/cytosolic phospholipase A2 cascade in hydroperoxide-stressed platelets. Free Radic Biol Med 35:616-625

17. Akkerman JW (2008) From low-density lipoprotein to platelet activation. Int J Biochem Cell Biol 40:2374-2378

18. Ferretti G, Rabini RA, Bacchetti T et al (2002) Glycated low density lipoproteins modify platelet properties: a compositional and functional study. J Clin Endocrinol Metab 87:2180-2184

19. Watanabe J, Wohltmann HJ, Klein RL, Colwell JA, Lopes-Virella MF (1988) Enhancement of platelet aggregation by low-density lipoproteins from IDDM patients. Diabetes 37:1652-1657

20. Calzada C, Coulon L, Halimi D et al (2007) In vitro glycoxidized low-density lipoproteins and low-density lipoproteins isolated from type 2 diabetic patients activate platelets via p38 mitogenactivated protein kinase. J Clin Endocrinol Metab 92:1961-1964

21. Grundy SM, Hansen B, Smith SC Jr, Cleeman JI, Kahn RA (2004) Clinical management of metabolic syndrome: report of the American Heart Association/National Heart, Lung, and Blood Institute/American Diabetes Association Conference on Scientific Issues Related to Management. Circulation 109:551-556

22. Havel RJ, Eder HA, Bragdon JH (1955) The distribution and chemical composition of ultracentrifugally separated lipoproteins in human serum. J Clin Invest 34:1345-1353

23. Bradford MM (1976) A rapid and sensitive method for the quantitation of microgram quantities of protein utilizing the principle of protein-dye binding. Anal Biochem 72:248-254

24. Browne RW, Armstrong D (2000) HPLC analysis of lipid-derived polyunsaturated fatty acid peroxidation products in oxidatively modified human plasma. Clin Chem 46:829-836

25. Therasse J, Lemonnier F (1987) Determination of plasma lipoperoxides by high-performance liquid chromatography. J Chromatogr 413:237-241

26. Lagarde M, Bryon PA, Guichardant M, Dechavanne M (1980) A simple and efficient method for platelet isolation from their plasma. Thromb Res 17:581-588

27. Born GV (1962) Aggregation of blood platelets by adenosine diphosphate and its reversal. Nature 194:927-929

28. Engelmann B, Brautigam C, Thiery J (1994) Plasmalogen phospholipids as potential protectors against lipid peroxidation of low density lipoproteins. Biochem Biophys Res Commun 204:1235-1242

29. Engelmann B, Schonthier UM, Richter WO, Duhm J (1992) Changes of membrane phospholipid composition of human erythrocytes in hyperlipidemias. II. Increases in distinct molecular species of phosphatidylethanolamine and phosphatidylcholine containing arachidonic acid. Biochim Biophys Acta 1165:38-44

30. Dreissigacker U, Suchy MT, Maassen N, Tsikas D (2010) Human plasma concentrations of malondialdehyde (MDA) and the F2isoprostane 15(S)-8-iso-PGF(2alpha) may be markedly compromised by hemolysis: evidence by GC-MS/MS and potential analytical and biological ramifications. Clin Biochem 43:159-167
31. Walsh SW, Vaughan JE, Wang Y, Roberts LJ 2nd (2000) Placental isoprostane is significantly increased in preeclampsia. FASEB $\mathrm{J}$ 14:1289-1296

32. Griesmacher A, Kindhauser M, Andert SE et al (1995) Enhanced serum levels of thiobarbituric-acid-reactive substances in diabetes mellitus. Am J Med 98:469-475

33. Garin MC, Kalix B, Morabia A, James RW (2005) Small, dense lipoprotein particles and reduced paraoxonase- 1 in patients with the metabolic syndrome. J Clin Endocrinol Metab 90:2264-2269

34. Gentile M, Panico S, Jossa F et al (2008) Small dense LDL particles and metabolic syndrome in a sample of middle-aged women. Findings from Progetto Atena. Clin Chim Acta 388:179 183

35. Tribble DL, Holl LG, Wood PD, Krauss RM (1992) Variations in oxidative susceptibility among six low density lipoprotein subfractions of differing density and particle size. Atherosclerosis 93:189-199

36. Ford ES, Mokdad AH, Giles WH, Brown DW (2003) The metabolic syndrome and antioxidant concentrations: findings from the Third National Health and Nutrition Examination Survey. Diabetes 52:2346-2352

37. Feillet-Coudray C, Rock E, Coudray C et al (1999) Lipid peroxidation and antioxidant status in experimental diabetes. Clin Chim Acta 284:31-43

38. Ford ES (2006) Intake and circulating concentrations of antioxidants in metabolic syndrome. Curr Atheroscler Rep 8:448-452

39. Hahnel D, Beyer K, Engelmann B (1999) Inhibition of peroxyl radical-mediated lipid oxidation by plasmalogen phospholipids and alpha-tocopherol. Free Radic Biol Med 27:1087-1094

40. Mallat Z, Lambeau G, Tedgui A (2010) Lipoprotein-associated and secreted phospholipases A in cardiovascular disease: roles as biological effectors and biomarkers. Circulation 122:2183-2200

41. Mattsson N, Magnussen CG, Ronnemaa T et al (2010) Metabolic syndrome and carotid intima-media thickness in young adults: roles of apolipoprotein B, apolipoprotein A-I, C-reactive protein, and secretory phospholipase A2: the cardiovascular risk in young Finns study. Arterioscler Thromb Vasc Biol 30:1861-1866

42. Borsch-Haubold AG, Kramer RM, Watson SP (1997) Phosphorylation and activation of cytosolic phospholipase A2 by $38-\mathrm{kDa}$ mitogen-activated protein kinase in collagen-stimulated human platelets. Eur J Biochem 245:751-759

43. Kramer RM, Sharp JD (1997) Structure, function and regulation of $\mathrm{Ca}^{2+}$-sensitive cytosolic phospholipase A2 (cPLA2). FEBS Lett 410:49-53

44. Calzada C, Vericel E, Lagarde M (1997) Low concentrations of lipid hydroperoxides prime human platelet aggregation specifically via cyclo-oxygenase activation. Biochem J 325:495-500

45. Podrez EA, Byzova TV, Febbraio M et al (2007) Platelet CD36 links hyperlipidemia, oxidant stress and a prothrombotic phenotype. Nat Med 13:1086-1095

46. Sattar N, Gaw A, Scherbakova O et al (2003) Metabolic syndrome with and without C-reactive protein as a predictor of coronary heart disease and diabetes in the West of Scotland Coronary Prevention Study. Circulation 108:414-419

47. Wiernsperger NF (2003) Oxidative stress as a therapeutic target in diabetes: revisiting the controversy. Diabetes Metab 29:579-585 